\title{
MAPPING THE RISKS OF MALARIA, DENGUE AND INFLUENZA USING SATELLITE DATA
}

\author{
R. K. Kiang ${ }^{\text {a }}$, R. P. Soebiyanto ${ }^{\text {b, a }}$ \\ a NASA Goddard Space Flight Center, Greenbelt, Maryland, USA - Richard.kiang@ @asa.gov \\ ${ }^{\mathrm{b}}$ Goddard Earth Sciences Technology \& Research, Universities Space Research Association, Columbia, Maryland, \\ USA - radina.p.soebiyanto@nasa.gov
}

Commission VIII, WG 2

KEY WORDS: Malaria, Dengue, Influenza, satellite, remote sensing

\begin{abstract}
:
It has long been recognized that environment and climate may affect the transmission of infectious diseases. The effects are most obvious for vector-borne infectious diseases, such as malaria and dengue, but less so for airborne and contact diseases, such as seasonal influenza. In this paper, we examined the meteorological and environmental parameters that influence the transmission of malaria, dengue and seasonal influenza. Remotely sensed parameters that provide such parameters were discussed. Both statistical and biologically inspired, processed based models can be used to model the transmission of these diseases utilizing the remotely sensed parameters as input. Examples were given for modelling malaria in Thailand, dengue in Indonesia, and seasonal influenza in Hong Kong.
\end{abstract}

\section{INTRODUCTION}

The transmission of infectious diseases is influenced by a myriad of factors. Environmental, meteorological, social, economic, political and warlike conditions have all been shown to contribute to the occurrence and outbreaks of a large number of diseases. Among these, the environmental and meteorological conditions are the factors that can be more easily quantified. They can be conveniently measured repeatedly using remote sensing in either friendly or hostile territories. Other factors, on the other hand, often require substantial efforts to measure and can only be expressed qualitatively.

Malaria is a parasitic disease that infects both humans and primates, and is endemic in most parts of the tropic, especially in the developing countries. Among the continents, Africa has nearly ninety per cent of the malaria cases and deaths. But malaria is also a significant problem in South and Southeast Asia. Malaria may still become a serious health issue for countries outside of the tropics where public health support is inadequate because of economic constraints or military conflicts. For example, since 1993 vivax malaria re-emerged in North Korea (Feighner 1998). Similarly, situated around $34^{\circ} \mathrm{N}$ with an arid climate, Afghanistan has approximately 0.41 to 0.6 million cases annually (Youssef 2008 WHO-EMRO 2007), and is the country most endemic with malaria within the World Health Organization's (WHO) Eastern Mediterranean Region. In this section, we will discuss malaria modelling and surveillance using remote sensing data. Examples are drawn from malaria in South Korea, Thailand, Indonesia and Afghanistan.

It is estimated that half of the world's population is at risk for malaria infection (RBM 2011). There are approximately 250 million cases annually with 0.9 million deaths worldwide. In recent years, the malaria burden has been significantly reduced through concerted efforts of international and national public health organizations, and the generous contributions from developed nations and philanthropic foundations. A major concern, however, is the emergence of artemisinin-resistant falciparum malaria that first appeared at the Thai-Cambodian border in 2007. Eliminating or containing the drug resistant strains is essential to global malaria control. Malaria is transmitted by infected female anophelines after taking blood meals from infectious humans. Five plasmodium species are known to infect humans, including Plasmodium vivax, $P$. falciparum, $P$. malariae, $P$. ovali, and the more recently discovered P. knowlesi (Singh 2004).

Similar to malaria, dengue fever and the more lethal dengue hemorrhagic fever are also mosquito-borne diseases. They are caused by flavivirus that is transmitted by infected Aedes mosquitoes. The latter may lead to the dangerous dengue shock syndrome with a mortality rate as high as $30 \%$. Two fifths of the world's populations are at risk. Dengue vectors can breed in any small amount of water such as vases, flower pots, discarded containers, or used tires. Hence it is challenging to implement larval control. Unlike malaria, dengue is considered an urban disease. Currently, dengue exists in Mexico and all Central American and Southeast Asian countries. There are four viral serotypes in the family of Flaviviridae.

Influenza is a common viral respiratory disease. Almost everyone gets infected one year or another. It infects five to fifteen per cent of the world population and causes 250,000300,000 deaths each year (WHO 2009). Despite vaccination and the largely mild cases, the burden of influenza remains significant due to health care cost and the loss of productivity. In the United States alone, the annual seasonal influenza epidemic can cause up to 200,000 hospitalization and more than 30,000 deaths (CDC 2010), the estimated economic burden based on the 2003 population is nearly US $\$ 90$ billion (Molinari 
et al., 2007). In addition to the significant burden of seasonal influenza, the persistent threat is that a pandemic causing strain may appear due to antigenic shift or reassortment.

There are three types of influenza virus circulating in the world: A, B and C. Type A and B are the most commonly found in humans. Type A is further classified into subtypes (e.g., H1N1) based on the types of hemagglutinin and neuraminidase on the surface of the virus.

In order to accommodate the ever changing circulating influenza strain, vaccine composition recommendations are updated twice a year. The recommendations are made by the World Health Organization (WHO) through its Global Influenza Surveillance Network (GISN).

\section{ENVIRONMENTAL DETERMINANTS}

\subsection{Malaria and Dengue}

Many factors are known to contribute to malaria transmission, including meteorological and environmental conditions, socioeconomic status, military conflicts and natural disasters. Among these, meteorological and environmental factors are perhaps the most noticeable. For example, malaria transmission may increase with the arrival or the end of a rainy season, and living near forest or water bodies may pose greater risk of mosquito bites and getting malaria. The El Niño-Southern Oscillation (ENSO) is a quasi-periodic climatic cycle that occurs every three to seven years across the tropical Pacific Ocean, and which causes excessive precipitation or droughts, has been shown to promote malaria transmission (Kovats 2003). Aside from precipitation, temperature and humidity are also important factors. Warmer temperatures hasten larval and vector development, and prolong mosquito life span and its consequent ability to transmit malaria. Warmer air retains more moisture and improves mosquito survivorship, so is higher humidity.

Vegetation has also been linked to malaria as it indicates the vector's breeding sites. For example, Anopheles dirus is a forest breeder, and An. maculates and An. sawadwongpori are rice field breeders. The Normalized Difference Vegetation Index (NDVI) (Tucker 1979) is one of the indices for vegetation condition. It is defined as the difference between the responses from the infrared and the red bands normalized by their mean. In modelling infectious diseases, NDVI is used most often to infer the precipitation which the area received before the satellite measurements were taken. The spatial distribution of NDVI can also be used to differentiate among urban, periurban, suburban and rural areas. Such information on the nature of the area is useful for malaria prevention and control.

Like malaria, temperature, humidity and rainfall are the important environmental determinants for dengue transmission. However, their effects on dengue transmission may be less obvious than those for malaria transmission because dengue vectors can breed indoor.

\subsection{Influenza}

The spatiotemporal variation of influenza across latitudes suggests that climate and environmental factors may have roles in influenza transmission and pathogenesis.
It is well known that influenza transmission in temperate climates is seasonal and peaks in the winter months. In the US, for example, influenza outbreaks often start as early as October, peak in February, and diminish by April or May; thus forming a distinct inter-annual oscillation pattern. In the tropics, there are significant influenza cases throughout the year, with one or two less distinct peak(s) whose timing varies geographically. It has been shown (e.g., Viboud et al., 2006) that influenza seasonal patterns vary with latitude, forming a traveling wave across the globe. Several studies that have explored the global migration pattern of influenza show varying travel pattern of influenza virus A migration out of the tropics and China; and migration between northern and southern hemispheres. Another study in Brazil showed that influenza starts in a low-population state near the equator during March-April, and travels southward towards temperate and more populous states (Alonso et al., 2007). Temperature, humidity and rainfall are among the factors that have been frequently implicated in influenza transmission.

\section{REMOTE SENSING MEASUREMENTS}

Satellite measurements of precipitation for estimating disease risks are most often derived from the Tropical Rainfall Monitoring Mission (TRMM) (Kummerow 1998). TRMM is a collaboration between the US and Japan. Japan built the precipitation radar (PR) and launched the spacecraft in 1999. There are five instruments on board: PR, TRMM microwave imager (TMI), Visible and Infrared Scanner (VIIRS), Cloud and Earth Radiation Energy Sensor (CERES), and Lighting Image Scanner (LIS). The main sensor that measures precipitation is TMI. Because the spatial resolution of the TRMM measurement is low, sometimes NDVI measured from a medium resolution satellite is used to infer the recent rainfall at a higher spatial resolution. Such inference is most effective for arid regions where little rainfall is received and vegetation growth is sensitive to rainfall, but less effective for regions with plenty rainfall. Land surface temperature and NDVI are both provided by the moderate resolution imaging spectroradiometer (MODIS). This sensor has thirty-six bands spanning the visible to the near-infrared wavelengths. Both the Terra and Aqua observatories are equipped with MODIS. NDVI, however, can be computed from any satellite instruments with red and infrared channels. However, because of the differences in band definitions, instrument characteristics, satellite orbits and measuring conditions, NDVI from different sensors must first be cross calibrated before they can be compared. In addition to the datasets described above, quite a few other satellites also provide data for ground cover classification, identification of potential larval habitats, and modelling malaria risks. For example, the well-known Landsat and SPOT series of satellites and the Advanced Spaceborne Thermal Emission and Reflection Radiometer (ASTER) and the Advanced Land Imager (ALI) (USGS 2009) are some of the multispectral sensors used for health monitoring purposes. Microwave sensors like Radarsat and the Phased Array L-band Synthetic Aperture Radar (PALSAR) are used over areas that are obscured frequently by clouds (JAXA 2011). The geoscience laser altimeter system (GLAS) is a light detection and ranging (LiDAR) sensor that is useful for differentiating vegetation types (NASA 2011). For high spatial resolution imagery, commercial data from IKONOS, QuickBird, or WorldView can be used (GeoEye 2011; DigitalGlobe 2011). 


\section{MODELING TECHNIQUES AND EXAMPLES}

Many factors including environmental and meteorological conditions affect the transmission of malaria, dengue and seasonal influenza. The environmental and meteorological conditions can indeed be considered the driving factors for these diseases when other factors are stable. This is especially evident for vector-borne diseases where the vector propagation is directly influenced by the environmental and meteorological conditions. This is the essential premise of why remote sensing can be used to predict disease risks. In general, statistical and biological models can be used to predict disease risks. Both types of models accept remotely sensed environmental and meteorological parameters as input. In some applications, remote sensing not only can be used for predicting risks, but also for detecting and reducing risks. In addition, remote sensing-based model can be used to project disease risk under the impact of global warming.

In general, statistical and mechanistic, or processed-based approaches are used to model disease risks based on satellite observations of meteorological and environmental parameters. In the statistical approach, epidemiological data are correlated with satellite data. The unknown parameters in the models are determined using statistical goodness of fit criteria, such as mean squared errors or Akaike Information Criteria (AIC). Once the model is trained, it can then be applied to other situations than those used for deriving the model parameters. How the pathogens actually transmit the disease under different meteorological and environmental conditions is not explicitly modelled in this approach. The common methods in this category include regression, time series analysis, and neural network. Examples are given in the following for using neural network to model malaria cases in Thailand and dengue cases in Indonesia; using Autoregressive Integrated Moving Average (ARIMA) to model influenza in Hong Kong; and using a discrete event simulation model to simulate malaria prevalence among 23 households.

\subsection{Malaria in Thailand}

Figure 1 shows an example for using neural network to predict malaria cases for the border provinces in Thailand (Kiang et al., 2006). The objective is to predict malaria cases in the near future in order to forewarn public health stakeholders on the expected transmission intensity. The main meteorological and environmental parameters used in modelling include precipitation, NDVI, and surface temperature. Excellent agreement between the actual and hindcast case rates is seen.

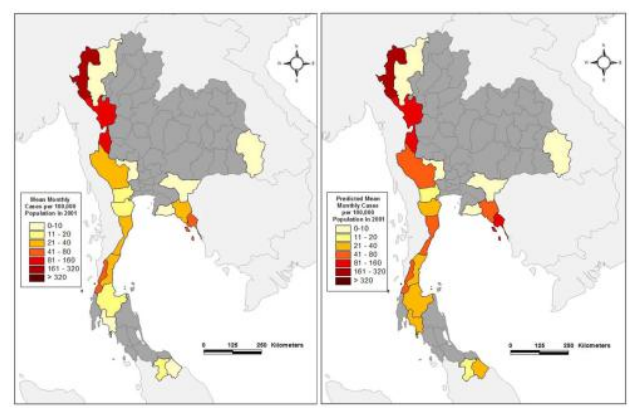

Figure 1. Actual (left) and predicted (right) malaria case rates in Thailand

\subsection{Dengue in Indonesia}

Figure 2 shows the results for using ARIMA to model the dengue cases in Jakarta, Indonesia using TRMM data and dew point temperature. All data except the last 12 months were used for the training. The last 12 months of data were used for deriving prediction accuracy. Close association between the actual and prediction distributions can be seen. Inconsistency between model output and the data at the final year is potentially due to the vector control effort that was implemented at the beginning of year 2005 and not accounted for in the model.

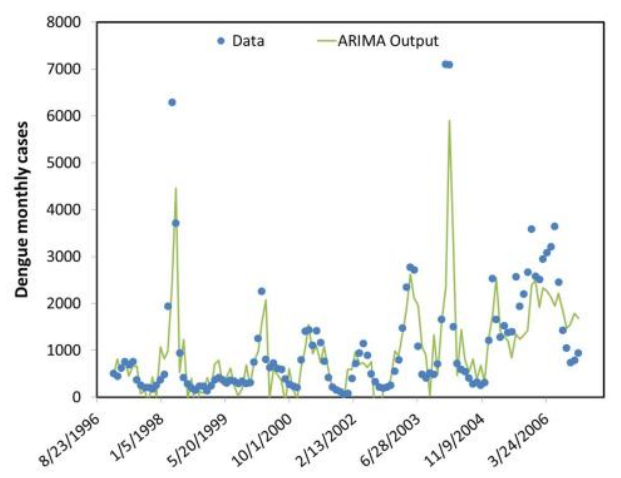

Figure 2. Modelled dengue cases in Jakarta, Indonesia

\subsection{Influenza in Hong Kong}

Figure 3 shows the results for using ARIMA and radial basis function neural network to model seasonal influenza cases in Hong Kong (Soebiyanto et al., 2010). Data from the last influenza season was used for testing modelling accuracy. The rest of the data were used for training. Input to these models include land surface temperature, rainfall, air and dew point temperature.

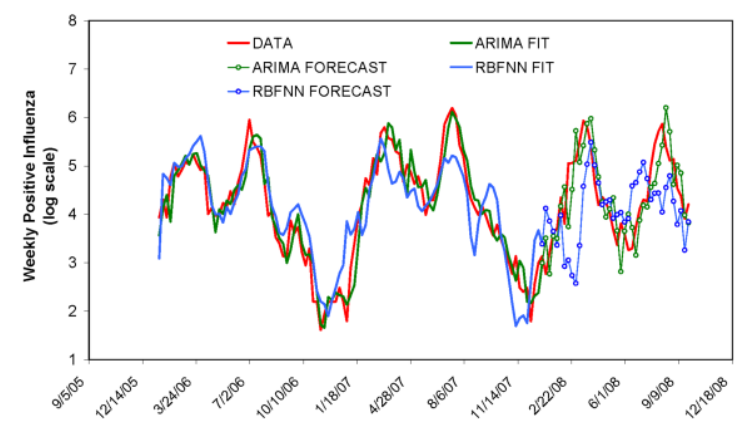

Figure 3. Modelled influenza cases in Hong Kong using ARIMA and radial basis function neural network

\subsection{Prevalence of Malaria in a Cluster of Households}

Figure 4 shows the expected malaria prevalence rate among 23 households and 92 residents using a discrete event simulation model. In this model, detailed interactions among vector life cycles, plasmodium sporogonic cycles, and human infection cycles were simulated under the influences of intrinsic and extrinsic effects. The simulations compare well with the shaded field measurements for vivax and falciparum malarias (Zollner, unpublished data). 


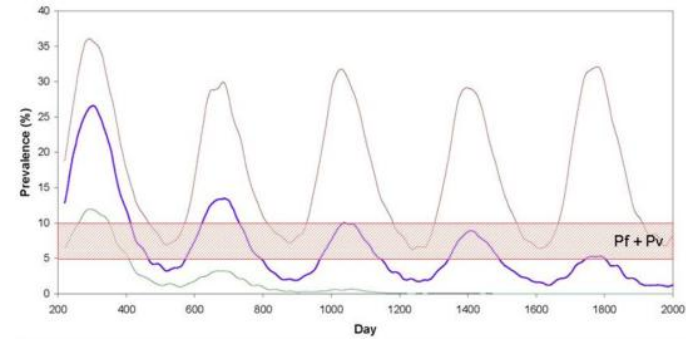

Figure 4. Simulated and observed malaria prevalence in a cluster of households

\section{CONCLUSION}

We have shown that remote sensing data can be used to model the risks for malaria, dengue, and seasonal influenza. These models can provide early warning and improve the response of public health organizations to these diseases.

\section{REFERENCES}

\section{References from Journals:}

Alonso, W.J., Viboud, C., Simonsen, L., Hirano, E.W., Daufenbach, L.Z. \& Miller, M.A. 2007. Seasonality of influenza in Brazil: a traveling wave from the Amazon to the subtropics. Amer. J Epidemiol. 165(12), pp. 1434-42.

Feighner, B.H., Pak, S.I., Novakoski, W.L. \& Kelsey, L.L. 1998. Reemergence of plasmodium vivax malaria in the Republic of Korea. Emer. Infect. Dis. 4(2), pp. 295-297.

Kiang, R., Adimi, F., Soika, V., Nigro, J., Singhasivanon, P., Sirichaisinthop, J., Leemingsawat, S., Apiwathnasorn, C. \& Looareesuwan, S. 2006. Meteorological, environmental remote sensing and neural network analysis of the epidemiology of malaria transmission in Thailand. Geospatial Health 1, pp.7184.

Kovats, R.S., Bouma, M.J., Hajat, S., Worrall, E. \& Haines, A. 2003. El Niño and health. Lancet 362. pp.1481-89.

Kummerow, C., Barnes, W., Kozu, T., Shiue, J. \& Simpson, J. 1998. The Tropical Rainfall Measuring Mission (TRMM) sensor Package. J. Atmos. \& Oceanic Tech. 15. pp.809-817.

Molinari, N.A., Ortega-Sanchez, I.R., Messonnier, M.L., Thompson, W.W., Wortley, P.M., Weintraub, E. \& Bridges, C.B. 2007. The annual impact of seasonal influenza in the US: Measuring disease burden and costs. Vaccine 25(27). pp.508696.

Singh, B., Sung, L.K., Matusop, A., Radhakrishnan, A., Shamsul, S.S., Cox-Singh, J., Thomas, A. \& Conway, D.J. 2004. A large focus of naturally acquired Plasmodium knowlesi infections in human beings. Lancet 363(9414). pp.1017-1024.

Soebiyanto, R.P., Adimi, F. \& Kiang, R.K. 2010. Modeling and predicting seasonal influenza transmission in warm regions using climatological parameters. PLOS ONE 5(3). e9450.

Tucker, C.J. 1979. Red and photographic infrared linear combinations for monitoring vegetation. Rem Sens. Environ. 8. pp.127-150.
Viboud, C., Alonso, W.J. \& Simonsen, L. 2006. Influenza in tropical regions. PLoS Med 3(4). e89.

Youssef, R., Safi, N., Hemeed, H., Sediqi, W., Naser, J.A. \& Butt, W. 2008. National malaria indicators assessment. Afghan. Ann. Malaria J. 1(1). pp.37-49.

\section{References from Books:}

Smith, J., 1989. Space Data from Earth Sciences. Elsevier, Amsterdam, pp. 321-332.

\section{References from Other Literature:}

WHO-Regional Office for the Eastern Mediterranean. 2007. Strategic plan for malaria control and elimination in the WHO Eastern Mediterranean Region 2006-2010. Cairo.

\section{References from websites:}

CDC, 2010. Key facts about seasonal influenza. http://www.cdc.gov/influenza/keyfacts.htm

DigitalGlobe Inc., 2011. QuickBird and WorldView. http://www.digitalglobe.com

GeoEye, 2011. Ikonos products and specifications. http://www.geoeye.com

JAXA. 2011. PALSAR.

http://www.eorc.jaxa.jp/ALOS/en/about/palsar.htm

NASA, 2011. ICESat. http://icesat.gsfc.nasa.gov

Roll Back Malaria, 2011. http://www.rbm.who.int

USGS, 2009. Earth Observing 1. http://eo1.usgs.gov

WHO, 2009. Influenza (Seasonal) Fact Sheet. http://www.who.int/mediacentre/fact sheets/fs211/en/

\section{ACKNOWLEDEMENTS}

This work was supported by NASA Applied Sciences Public Health Program and CDC Influenza Division. 